\section{Nivel de conocimientos en salud bucal de las madres y su relación con el estado de salud bucal del niño menor de cinco años de edad}

Level of knowledge on oral health of mothers and their relation to the oral health status of children under five years of age

\begin{abstract}
Resumen
El objetivo de este estudio fue determinar el nivel de conocimientos sobre salud bucal de las madres de familia y su relacion con el estado de salud bucal del nińo menor de cinco ańos de edad, que acude a establecimientos hospitalarios del Ministerio de Salud (MINSA) y Seguro Social (ESSALUD), teniendo en consideración los criterios y normas éticas establecidas; siendo un estudio de tipo analítico transversal. La muestra estuvo constituida por madres con sus hijos menores de cinco años de edad (n 143). El tipo de muestreo fue no probabilística por cuotas. Se aplicó una encuesta estructurada para la recolección de información del nivel de conocimientos sobre salud bucal de las madres de familia y ficha de diagnóstico del estado de la salud bucal del nińo menor de cinco años, que fueron examinados clínicamente a luz artificial, utilizando los índices epidemiológicos de: Caries Dental (ceo-d), Placa bacteriana (Greene y Vermillion. IHO-S). No se halló asociacion significativa entre el nivel de conocimientos sobre salud bucal que poseen las madres, con el estado de salud bucal del nińo menor de cinco años de edad (p 0,16 ). Si bien las madres poseen conocimientos entre regular y bueno en un $77.6 \%$, estos no son aplicados adecuadamente, por lo que se debe trabajar dando énfasis en todo programa preventivo promocional para propiciar actitudes positivas hacia el cuidado de la salud bucal, desde temprana edad, resaltando el rol que cumple la dentición temporal, no solo para garantizar el espacio de los dientes permanentes, sino mucho más como es la alimentación del niño y el rol que juega para su buen crecimiento y desarrollo bio-psico-social, logrando así estilos de vida adecuados.
\end{abstract}

Palabras claves: salud bucal, caries dental, higiene bucal

\begin{abstract}
The aim of this study was to determine the level of knowledge on oral health of mothers and their relationship with oral health status of their children under five years old, who were admitted to hospitals of Ministry of Health (MINSA) and Social Security (ESSALUD). Were taken into consideration ethical standards. This is an analytical and cross-sectional study. The sample consisted of mothers with children under five years of age (n 143). Was used not randomized quota sampling. Was applied a structured questionnaire to collect information about the level of mother's knowledge on oral health and a diagnostic sheet for oral health status of children under five. Clinical examination was performed with artifitial light, using epidemiological indices: Dental Caries Index (dmft), and the Simplified Oral Hygiene Index by Greene-Vermillion (OHI-S). There was no significant association between level of knowledge on oral health who have mothers with the oral health status of children under five years of age ( $\mathrm{p} 0.16$ ). Mothers had knowledge among regular and good in $77.6 \%$ however this was not reflected in the oral health of children. Was concluded that it should work a program with emphasis on promoting preventive promotional positive attitudes towards oral health care from an early age not only to ensure the spacing of permanent teeth, but also to ensure the child's feeding and their growth and bio-psycho-social development.
\end{abstract}

Keywords: oral health, dental caries index, oral hygiene index
Artículo Original

Lourdes A. Benavente Lipa ${ }^{1}$,

Sylvia A. Chein VIllacampa ${ }^{1}$,

Carlos H. Campodónico

Reátegui', Elmo Palacios

Alva ${ }^{2}$, María S. Ventocilla

Huasupoma ${ }^{3}$, Antonia Castro

Rodríguez ${ }^{1}$, Olinda Huapaya

Paricoto², María A. Álvarez

Paucar ${ }^{4}$, Wilder Paulino

Córdova ${ }^{5}$, Antonio Espetia

Aguirre $^{5}$

1 Profesores del Departamento Académico Estomatología Preventiva y Social

2 Profesores del Departamento Académico Estomatología Médico Quirúrgico

3 Profesora del Departamento Académico Estomatología Rehabilitadora

4 Profesores del Departamento Académico Estomatología Pediátrica

5 Internos de la Facultad de OdontologíaUNMSM.

Correspondencia:

MG. Lourdes A. Benavente Lipa.

Facultad de Odontología UNMSM. Av. Germán Amézaga s/n, Lima, 1 Perú.

Correo electrónico: lbenaventel@yahoo.com

Fecha de recepción: 28-02-12

Fecha de aceptación: 16-05-12

\section{Introducción}

Consideramos que la madre adquiere gran relevancia y puede constituirse en un factor de riesgo del estado de salud de su hijo conllevando esto a un problema familiar, social, económico y educacional. La Educación para la Sa- lud es uno de los ejes que aseguran la preservación de salud integral del niño, asegurando así una persona con una mejor calidad de vida. ${ }^{1,2,3,4}$ Es el conocimiento de los principales factores de riesgo de su salud bucal en nuestra realidad. 5,6,7 Servirá para implementar y consolidar futuros programas de pro- moción y prevención de salud bucal, las cuales deben ser con una metodología participativa (intervención-acción), para poder generar un impacto efectivo y así controlar los principales factores de riesgo desde la infancia temprana, con el fin de disminuir la morbilidad bucal, promoviendo estilos de vida sa- 
ludables desde el entorno familiar. ${ }^{2,3}$, $4,7,8$ Los padres de familia son los responsables de la Educación para la Salud Bucal en los hogares, pero no todos están preparados para poder realizarla correctamente, ya que muchos transmiten a sus hijos la cultura que ellos recibieron, con conceptos y hábitos equivocados, muchas veces perjudiciales para el nińo. ${ }^{9,10,11}$

El objetivo de este estudio es determinar el nivel de conocimientos sobre salud bucal de las madres de familia y su relación con el estado de salud bucal del niño menor de 5 años de edad, que acude a establecimientos hospitalarios del MINSA y ESSALUD.

Esta investigación pretende convertirse en un referente básico, ya que este tema es poco estudiado y no hay mucha literatura publicada en nuestro medio. Se espera que el esfuerzo realizado se vea compensado con el interés que otros investigadores pongan en esta área. La meta es producir un conocimiento útil que pueda aplicarse para diseñar protocolos de promoción de la salud bucal, para lograr la meta de salud de la OMS, de tener para el ańo 2020, niños menores de 5 años con cero caries ${ }^{12}$. Por tanto se considera importante brindar educación para lograr que la comunidad tenga una "Cultura de Salud" temprana, oportuna y permanente dirigida a la madre en aspectos de cuidados de la salud bucal de sus hijos, solo así tendremos asegurados niños con niveles de crecimiento y desarrollo óptimos, para competir en el mundo globalizado de hoy; lo que por otro lado demandará al estado y a la sociedad un mejor costobeneficio.

\section{Materiales y método}

Esta investigación es de tipo analítico y transversal. La población estuvo constituida por madres de familia con sus hijos menores de 5 años de edad, (6 a 60 meses), que acuden a establecimientos hospitalarios del MINSA y de ESSALUD, realizado en el Departamento de Lima-Perú, durante el periodo de agosto y setiembre del 2010. La muestra estuvo constituida por madres con sus hijos menores de 5 años de edad $(n=143)$. El tipo de muestreo fue no probabilístico por cuotas. Los criterios de inclusión fueron, niños con aparente buen estado biológico y psicológico, que no estén recibiendo ningún tratamiento de tipo preventivo-promocional odontológico, cuyas madres no hallan recibido en el presente año algun tipo de educación en aspectos de salud bucal. Se inclu- yeron en el estudio las madres con sus hijos menores de 5 años, que aceptaron participar, firmando el consentimiento informado. La muestra fue equitativa en ambos instituciones de salud. Para la recolección de información primero se aplicó a las madres una encuesta estructurada, anotando su edad, grado de instrucción, ocupación y lugar de atención (MINSA o ESSALUD). Luego se recabaron los datos de conocimientos que poseen estas; con 20 preguntas, sobre conocimientos de la salud bucal del niño menor de 5 ańos: partes de la boca, dientes, funciones, factores de riesgo y medidas preventivas, dieta, consumo de medicamentos, uso del biberón, lactancia, chupón, higiene bucal, usos, funciones y aplicación de flúor, sellantes, asistencia al consultorio dental, periodicidad según edad del niño: Asignándole un puntaje a cada pregunta de: 1 bajo, 2 regular, 3 bueno. Se catalogaron en tres niveles de conocimientos según puntaje obtenido: < 33 bajo, 34-47 regular, 48-60 bueno. Así mismo se preparó una ficha de diagnóstico para el niño, obteniendo su edad en meses, género. Para la evaluación del estado de salud bucal del niño, se utilizaron los índices de: Caries Dental (ceo-d: sano=0, bajo=1 a 3, mo-

\section{Resultados}

La edad de los niños estuvo entre los 6 a 60 meses, siendo el $44 \%$ los de 37 a 60 meses. El $60 \%$ de las madres del estudio tiene educación secundaria. De las cuales el $72 \%$ son ama de casa. y el 68,5\% de ellas poseen un nivel de conocimiento regular sobre salud bucal. (Ver Tabla 1 ).

Tabla 1. Caracterización de la población estudiada

\begin{tabular}{lc}
\hline Edad de los niños (meses) & $\%$ \\
\hline $6-18$ & 19,6 \\
$19-36$ & 36,4 \\
$37-60$ & 44,0 \\
\hline Grado de instrucción de las madres & \\
\hline Primaria & 7 \\
Secundaria & 60 \\
Superior técnica & 22 \\
Superior universitaria & 11 \\
\hline Ocupación de las madres & \\
\hline Profesional & 7,7 \\
Obrera & 5,6 \\
Empleada & 4,9 \\
Su casa & 72,0 \\
Independiente & 8,4 \\
Otro & 1,4 \\
\hline Nivel de conocimiento de las madres & \\
\hline Bueno & 9,1 \\
Regular & 68,5 \\
Bajo & 22,4 \\
\hline
\end{tabular}

derado $=4$ a 5 , alto $=6$ a más) incluyendo la detección de lesiones incipientes como la mancha blanca, la Placa bacteriana (IHO simplificado de Grenee y Vermillon-modificado de placa blanda) de la Organización Mundial de la Salud (OMS). Estos instrumentos fueron validados en un estudio piloto con un grupo de madres con niños menores de 5 años con características similares, haciéndose los ajustes respectivos.

Para la toma de datos, primero se aplicó la encuesta a la madre, luego se realizó evaluación clínica del estado de salud bucal del nińo menor de 5 años de edad en la consulta externa de Control, crecimiento y desarrollo CRED- del nińo de los establecimientos de salud, sentados en una silla y con luz artificial (fronto luz).

Para establecer asociación entre el Nivel de conocimientos sobre salud bucal estado de salud bucal del niño menor de 5 años de edad, se usó la prueba Chi Cuadrado, asumiendo un límite de valor p 0.05 para establecer la significancia. Para el análisis estadístico se utilizó el Software SPSS versión 15. de las madres de familia con la variable 
El $16.8 \%$ de los nińos menores de 18 meses tiene dientes sanos y el $17.5 \%$ de los niños mayores de 37 meses tiene un índice alto de caries dental; existiendo asociación significativa $(\mathrm{p}=0,00)$. (Ver Fig. 1).

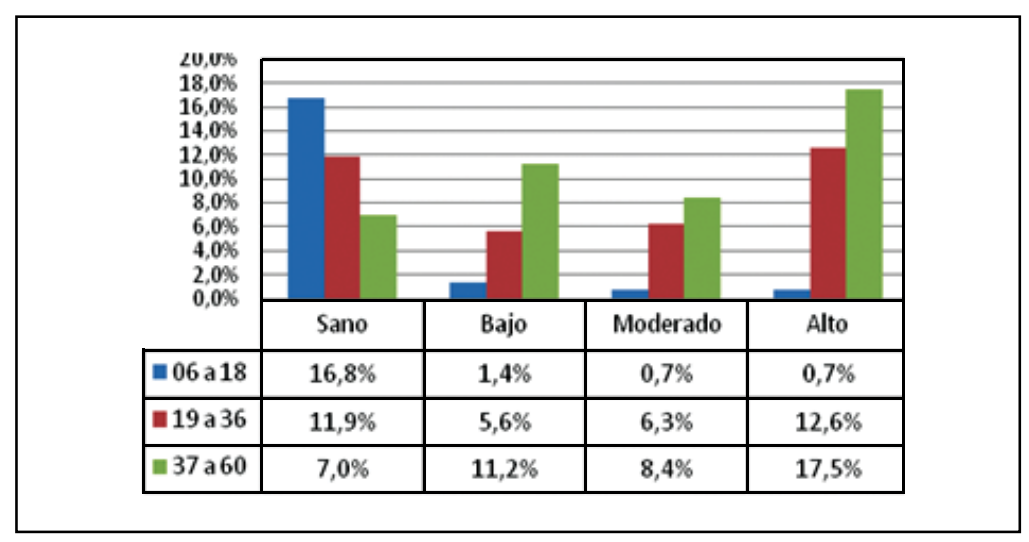

Fig 1. Caries dental según edad de los niños.

Se encontró asociación significativa entre caries dental e higiene bucal en los niños del estudio ( $p=0,00)$. (Ver Fig. 2)

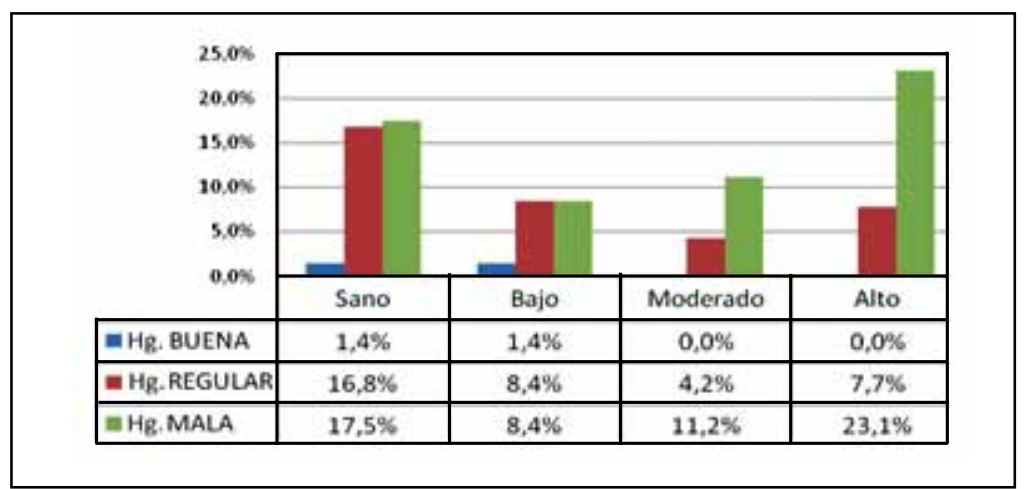

Fig 2. Caries dental e higiene bucal de los niños

No existe asociación significativa entre nivel conocimiento de las madres de familia y la frecuencia de caries dental de sus hijos menores de 5 ańos de edad $(p=0,15)$. (Ver Fig. 3).

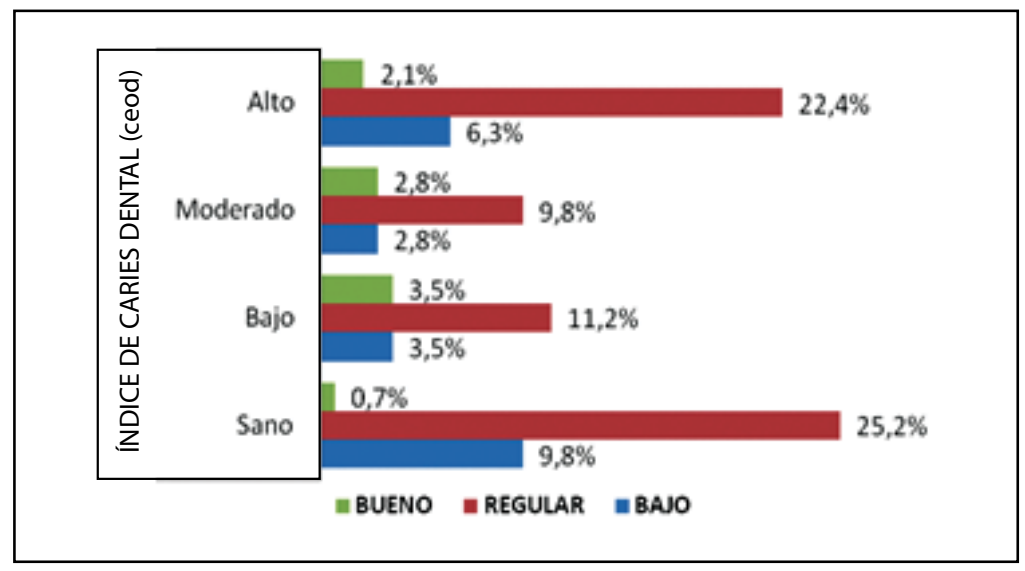

Fig. 3. Nivel de conocimiento de las madres y caries dental de los niños

Respecto al nivel conocimiento de las madres de familia, no existe asociación significativa, en relación a la higiene bucal de sus hijos menores de 5 años de edad del estudio ( $\mathrm{p}=0,17)$. (Ver Fig. 4).
Existe asociación significativa entre el grado de instrucción y nivel conocimiento de las madres de familia $(\mathrm{p}=0,02)$. (Ver Fig. 5).

Respecto al establecimiento asistencial de salud, existe diferencia estadísticamente significativa, en cuanto a la frecuencia de caries que presentan los nińos del estudio ( $\mathrm{p}=0,00)$. (Ver Fig. 6).

Los niños del estudio presentaron una media de tres dientes afectados por caries dental, lo que significa que en promedio tienen un Índice de Caries Moderado; 13 dientes sanos y 2,7 dientes no erupcionados, así como un Índice Higiene Bucal de 1,9 (malo). En cuanto al puntaje promedio de conocimientos de sus madres fue de 37,8 (regular). (Ver Tabla 2).

\section{Discusión}

La salud bucal es un constituyente inseparable e importante de la salud general ya que las enfermedades del sistema estomatognático afectan a la salud general del individuo y tienen consecuencias fisiológicas aún más complejas, porque pueden llegar a afectar la nutrición, las relaciones interpersonales y hasta la salud mental de la persona. Lo fundamental es orientar hábitos adecuados de higiene, alimentación, etc., así como factores que favorecen la aparición de enfermedades bucales. Los resultados obtenidos se consideran importantes, ya que para que una intervención tenga éxito es necesario conocer previamente las necesidades reales del grupo poblacional al cual va dirigida, tratando de enfocarse en los aspectos que influyen en la naturaleza misma de la enfermedad, los cuales pueden ser analizados desde la perspectiva de los padres para servir de guía a los profesionales y tenerlos en cuenta para preservar la salud bucal en estos individuos, tal como lo afirman Martignon $S$ y col. ${ }^{13}$ y Franco A y col. ${ }^{14}$

Coincidimos totalmente con Walter L y col. ${ }^{15}$, que reportaron que a medida que aumenta la edad del niño, aumenta también la frecuencia de caries.

Se encontró que prevalece el grado de instrucción secundaria de las madres (60 \%) y que existe asociación significativa entre el grado de instrucción y nivel conocimiento de las madres de familia, coincidiendo así con Velásquez $Z^{16}$ que halló que el nivel de conocimientos fue regular en el 60,6 \% de madres gestantes y que existe un estrecha relación entre el nivel de conocimientos y el grado de instrucción. 


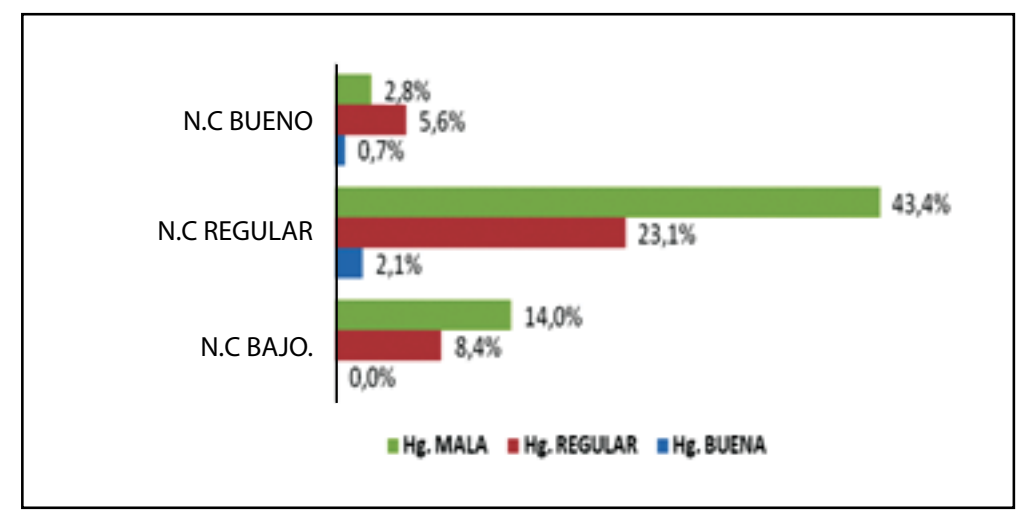

Fig. 4. Nivel conocimiento de las madres e higiene bucal de los niños.

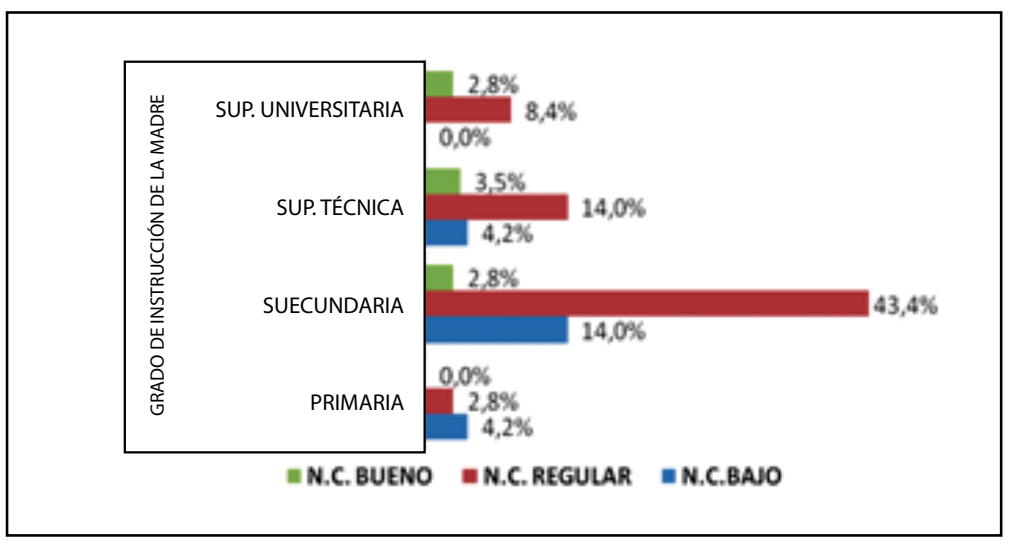

Fig. 5. Nivel de conocimientos de las madres según su grado de instrucción.

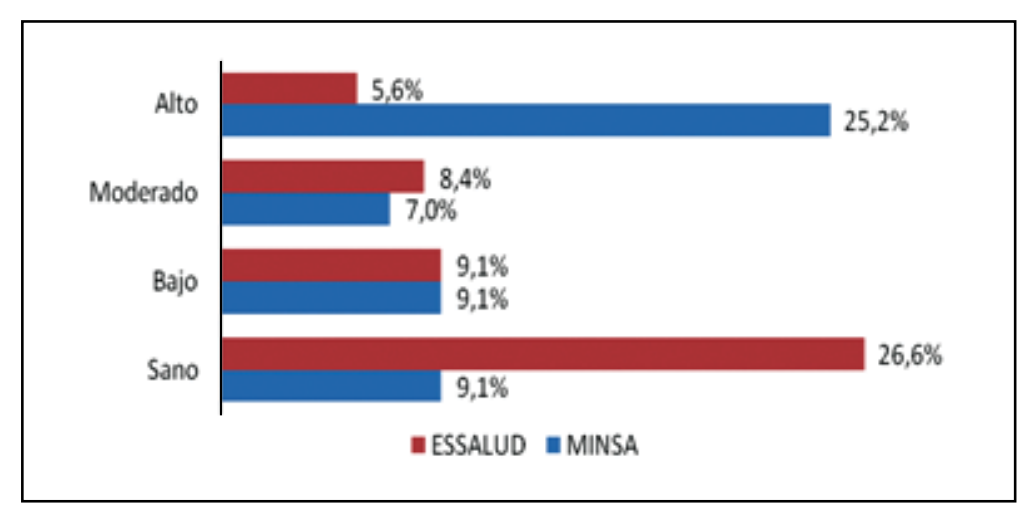

Fig. 6. Caries dental de los niños y el establecimiento de salud donde acuden.

Tabla 2. Estado de salud dental e higiene bucal de los niños y puntaje obtenido en conocimientos por las madres

\begin{tabular}{|c|c|c|c|c|c|c|c|c|}
\hline \multirow{2}{*}{ 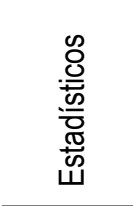 } & \multicolumn{4}{|c|}{ Estado de dentición temporal (ceod) } & \multirow{2}{*}{$\begin{array}{l}\text { Diente } \\
\text { No Erup- } \\
\text { cionado }\end{array}$} & \multirow{2}{*}{$\begin{array}{c}\text { Mancha } \\
\text { Blanca }\end{array}$} & \multirow{2}{*}{$\begin{array}{c}\text { Higiene } \\
\text { Bucal } \\
\text { de los } \\
\text { Niños }\end{array}$} & \multirow{2}{*}{$\begin{array}{c}\text { Puntaje } \\
\text { Obtenido } \\
\text { por las } \\
\text { Madres }\end{array}$} \\
\hline & $\begin{array}{l}\text { Diente } \\
\text { Cariado }\end{array}$ & $\begin{array}{c}\text { Diente con } \\
\text { Extracción } \\
\text { Indicada }\end{array}$ & $\begin{array}{c}\text { Diente } \\
\text { Obturado }\end{array}$ & $\begin{array}{c}\text { Diente } \\
\text { Sano }\end{array}$ & & & & \\
\hline Media & 3,0 & 0,1 & 0,2 & 13,1 & 2,7 & 0,6 & 1,9 & 37,8 \\
\hline Desv. St. & 4,0 & 0,7 & 0,9 & 5,2 & 4,7 & 1,4 & 0,6 & 8,4 \\
\hline
\end{tabular}

La Torre $\mathrm{GM}^{17}$ halló que el 85 \% de madres gestantes tiene bajo conocimiento sobre el cuidado de la salud bucal de los niños menores de 5 años, muy lejos de lo que encontramos en el presente, donde el $68.5 \%$ de las madres tiene un nivel conocimientos regular sobre salud bucal.

En esta investigación hallamos que las madres con características sociales media baja, poseen un nivel de conocimientos de regular (68.5\%) y bueno $(9.1 \%)$, lo que no se ve reflejado en el estado de salud bucal del niño, por lo que se presume, que si bien las madres poseen conocimientos entre regular y bueno en un $77.6 \%$, estos no son aplicados adecuadamente, por lo que se debe trabajar dando mucho énfasis en todo programa preventivo promocional para instalar actitudes positivas hacia el cuidado de la salud bucal, desde temprana edad, resaltando el rol que cumple la dentadura temporal, no solo para garantizar el espacio de los dientes permanentes, sino mucho más para la alimentación del niño y el rol que juega esto para su buen crecimiento y desarrollo bio-psico-social, instalando así estilos de vida adecuados. En este sentido Martignon S y col. ${ }^{13}$, encuentran que las madres de niños menores de 5 años, poseen bajo conocimiento sobre etiología, prevención y actitudes frente a la higiene y alimentación del niño, siendo mayor en familias de clase social baja.

No se halló que existe asociación significativa entre el nivel de conocimientos sobre salud bucal que poseen las madres de familia con el estado de salud bucal del niño menor de 5 ańos de edad, tal como Sosa ${ }^{18}$ en su estudio demuestra que las madres, dicen una cosa y al momento de observarlas es muy diferente a lo que manifiestan, es decir, no siempre el conocimiento está relacionado con la actitud o prácticas que se realizan. Se halló asociación significativa de la frecuencia de caries dental con género, siendo el masculino el más afectado, demostrando relación entre el índice de caries e higiene, así mismo cuando se relacionó con los establecimientos donde acuden a recibir atención, se observó que los niños de ESSALUD (26.6 $\%)$ tienen buen estado de salud bucal, mientras que en MINSA el $25.2 \%$ de niños tiene alto índice de caries dental. Finalmente los resultados del estudio serán aplicables para el diseño de intervenciones acordes con las necesidades de los individuos, lo que garantizará un impacto positivo sobre la salud bucal, a partir de la consolidación de los conocimientos, promoción de actitudes favo- 
rables y prácticas de cuidado adecuadas. Siendo la salud bucal un aspecto importante en el cuidado integral de la población infantil, las madres de familia se consideran la fuente primaria de la educación temprana en los menores, así como los maestros y toda persona encargada de su cuidado ${ }^{16,19}$.

\section{Conclusiones}

Las madres de familia tienen un nivel de conocimiento regular sobre salud bucal, sin embargo la salud bucal de los hijos de las madres encuestadas es mala, lo que revela ausencia de asociación entre el nivel de conocimientos de salud bucal que poseen las madres, con el estado de salud bucal de sus hijos menores de 05 años en la población estudiada

\section{Agradecimiento}

Al Consejo Superior de Investigación de la UNMSM, por el financiamiento al presente estudio.

\section{Referencias bibliográficas}

1. Gonzales R. Educación de la madre y el estado de salud de las primeras molares permanentes en sus hijos de 6-10 años. Lima-Perú. [Tesis de Bachiller] Lima. Facultad de Odontología, Universidad Nacional Mayor de San Marcos. 2000

2. Arias C. Nivel de conocimiento sobre prevención de salud Oral en gestantes que acuden a 3 centros de salud de Lima de dic. 95 a ene. 96. [Tesis de Bachiller] Lima. Facultad de Odontología, Universidad Nacional Federico Villarreal. 2000.

3. Benavente L. Influencia del nivel de educación sanitaria de los padres o apoderados en el estado de salud e higiene bucal del niño con retardo mental. [Tesis de Maestría] Lima. Facultad de Odontología. Universidad Nacional Mayor de San Marcos. 2005.
4. Díaz M. Grado de conocimiento de medidas preventivas de Salud Bucal en gestantes. [Tesis de Bachiller] Lima. Facultad de Odontología, Universidad Peruana Cayetano Heredia. 1996.

5. Holdings LLC. Investigaciones sobre el riesgo cariogénico durante el primer año de vida del bebé. Acta Odontológica Venezolana. 2000:16-18

6. PRECONC Programa de Educación Continua Odontológica No Convencional/ OPS-OMS. "Odontología Preventiva" Bordoni N. Washington D.C. 1993:24-30

7. Tello MG. Estudio epidemiológico de la prevalencia de caries y su relación con hábitos alimentarios y de higiene bucal en niños de 6 a 36 meses. Lima-Perú. [Tesis de Titulo Profesional] Lima. Universidad Inca Garcilaso de la Vega. 2001

8. Navas R, Álvarez C, Rojas-Morales, T, Zambrano O. Metodología estratégica para la participación de los padres en los cuidados de la salud bucal de niños preescolares. Rev. Ciencia Odontológica 2005:26-28

9. Montellano G, Brieke W, Cáceres $S$, Orduña C, Serrano S. Factores de riesgo en pacientes con caries temprana de la infancia del departamento de estomatología del hospital para el niño poblano. Revista Oral 2004; 5:230-232.

10. Cuartas J, Alvear A. Relación entre percepción de los padres sobre el tratamiento odontológico y sus hábitos de higiene oral, con la historia de caries dental en sus hijos, entre 3 y 5 años de edad. Revista Ces. Odontología 2002; 15(1):13-18.

11. Contreras N, Valdivieso M, Cabello E. Nivel de conocimientos y prácticas de medidas preventivas de profesionales de salud sobre caries dental en el infante. Rev. Estomatológica Herediana. 2008; 18(1):29-34.
12. OMS/ FDI. Metas Globales para la Salud Bucal en el año 2000. Revista Salud Bucal CORA (Confederación Odontológica de la República Argentina). Año VIII (48) Dic 1981.

13. Martignon S, González M, Jácome S, Velosa J, Santamaría R. Conocimientos, actitudes y prácticas en salud oral de padres y jardineras de niños hogares infantiles: ICBF - Usaquén, Bogotá. Revista científica Universidad El Bosque 2003; 9 (2):2003:24-27

14. Franco A, Santamaría A, Kurzer E, Castro L, Giraldo M. El menor de seis años: situación de caries y conocimientos y prácticas de cuidado bucal de sus madres. Revista CES Odontología 2004; 17 (1):19-29.

15. Walter L y col. Odontología para bebé: Odontopediatría del nacimiento a los 3 ańos. S.P. Edit. Artes Médicas. 2000:45-50, 64-67

16. Velásquez Z. Nivel de conocimiento sobre medidas preventivas de enfermedades bucales en un grupo de madres gestantes del Instituto Peruano de Seguridad Social. [Tesis de Bachiller] Lima. Universidad Peruana Cayetano Heredia. 1995.

17. La Torre GM. Gestantes primíparas y conocimiento del cuidado de salud Bucal infantil en el Hospital $\mathrm{Na}$ cional Hipólito Unanue. [Tesis de Bachiller] Lima. Universidad Particular San Martín de Porras. 2001.

18. Sosa L. Motivación en salud bucal de las madres en el área de hospitalización pediátrica Hospital General Victorino Santaella Ruiz. Disponible en: www.odontologia-online. com//Ciencia_bucal. Consultado el 23-02-2010

19. Duque de Estrada RJ, Rodríguez CA, Couting, Riveron HF. Factores de riesgo asociados con la enfermedad caries dental en niños. $\mathrm{Rev} \mathrm{Cu}$ bana Estomatol, 2003 mayo-ago; 40(2) [revista en internet] Disponible en : http://scielo.sld.cu/ 